\title{
Line Planning on Path Networks with Application to the Istanbul Metrobüs
}

\author{
Ralf Borndörfer, Oytun Arslan, Ziena Elijazyfer, Hakan Güler, Malte Renken, \\ Güvenç Şahin, and Thomas Schlechte
}

\begin{abstract}
Bus rapid transit systems in developing and newly industrialized countries often consist of a trunk with a path topology. On this trunk, several overlapping lines are operated which provide direct connections. The demand varies heavily over the day, with morning and afternoon peaks typically in reverse directions. The construction of a line plan for such a system can be seen as a multi-period covering problem on an interval graph. We propose an integer programming model for this problem, derive a structural property of line plans in the static (or single period) "unimodal demand" case, and consider approaches to the solution of the multi-period version that rely on clustering the demand into peak and off-peak service periods. An application to the Metrobüs system of Istanbul is discussed.
\end{abstract}

\section{Introduction}

The establishment of a public transportation system involves decision making on network design, line planning, timetabling, and fare planning. Due to the (contradictory) objectives of minimizing the costs and maximizing the level of service, each

Ralf Borndörfer, Ziena Elijazyfer, Malte Renken, Thomas Schlechte

Zuse Institute Berlin, Takustr. 7, 14195 Berlin, e-mail: \{surname \} @ zib. de

Oytun Arslan

IVU Traffic Technologies AG, Borchersstr. 20, 52072 Aachen e-mail: oar@ivu.de

Hakan Güler

Sakarya University, Engineering Faculty, Civil Engineering Department, Transportation Engineering Division, 54187 Esentepe/Sakarya e-mail: hguler@sakarya.edu.tr

Güvenç Şahin

Sabanci University, Industrial Engineering, 34956 Orhanli Tuzla/Istanbul e-mail: guvencs @ sabanciuniv.edu 
task is already challenging on its own, such that the planning process is typically conducted in succession [1]. Its integrated treatment has been taken up only recently [2], and it is still quite unclear how a "globally optimal system" should look like or how it could be identified. The investigation of basic, but practically relevant, classes of networks is one way to advance in this direction.

We consider an interesting type of transportation systems with the simplest possible network structure: a path topology is often found in bus rapid transit (BRT) systems in developing or newly industrialized countries, such as Trolébus in Quito [5], or our subject of investigation, the Metrobüs in Istanbul, see Fig. 1. Even though such systems may, at first sight, look small in terms of numbers of stations or links, they typically service the bulk of the demand, exerting a trunk function.

In BRT systems such as Metrobüs, the demand is typically highly asymmetric w.r.t. its distribution on the line and notably fluctuating w.r.t. time: There is a morning peak towards the center, an evening peak towards the outskirts, and significantly less demand during other times, see Fig. 2. Traditional line planning addresses this demand fluctuation by constructing a base service, which is augmented in peak hours, or vice versa. But is this prevalent procedure conclusive? This is the question that we study in this article. In fact, as far as we know, multiperiod line planning (much less time continuous) has not been considered in the optimization literature so far.

The paper studies the line planning problem on path networks over a planning horizon of an entire day. In a path network, the passenger travel paths are uniquely determined. This gives rise to a fixed passenger load (or volume) on every individual link of the path network. These loads have to be covered by lines that must offer appropriate capacities and frequencies. We concentrate on the two dominating features of the demand characteristics of the problem: (i) asymmetricity of the demand towards the center of the network creates a unimodal (increasing to a maximum and then decreasing) demand distribution along the path, (ii) the level of difference in the total number of passengers between peak and off-peak hours requires partitioning the day into multiple sections. We show that unimodal demand leads to a unimodal line plan in the single period case. This gives an indication that line plans that augment a base service in peak hours might work well, i.e., that augmentations in space and time are reasonable. A computational comparison of two solutions approaches for the multiperiod case on Metrobüs data corroborates this claim.

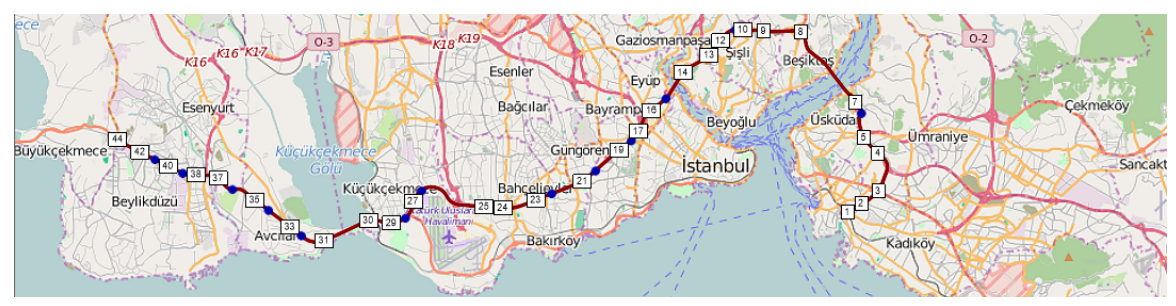

Fig. 1 The Metrobüs in Istanbul with its 44 stations (visualization by PTV Visum [4]). 


\section{The Static Demand Case}

We study the following demand coverage model to assign frequencies to a given set of lines, that are modeled as paths on a traffic network, which in our setting itself has path topology, in the static demand or single period case. Consider a traffic network $N=\left(V=[n], A=A^{\rightarrow} \cup A^{\leftarrow}\right)$ as a path with forward $\operatorname{arcs} A^{\rightarrow}=\{(i, i+1): i \in[n-1]\}$ and backward arcs $A^{\leftarrow}=\{(i+1, i): i \in[n-1]\}$. Let $c: A \rightarrow \mathbb{R}^{+}$be a cost and $d$ : $A \rightarrow \mathbb{R}_{0}^{+}$the demand, i.e., the number of passengers traveling across each arc. Let the set of lines $L$ consist of all non-trivial (directed) paths on $N$ with endpoints in some set $T=\left\{t_{1}, \ldots, t_{m}\right\} \subseteq V$ of terminal stations, $t_{1}<\cdots<t_{m}$, and denote the beginning and end of line $l \in L$ by $\alpha(l)$ and $\omega(l)$. It is reasonable to require the beginning and end of $P$ to be in $T$, i.e., $t_{1}=1$ and $t_{m}=n$. A function $p: L \rightarrow \mathbb{N}_{0}$ is called a line plan, and $p(l)$ is the frequency of line $l$. We say that a line plane is balanced if the flow balance conditions $\sum_{l \in L: \alpha(l)=v} p(l)=\sum_{l \in L: \omega(l)=v} p(l)$ hold for every vertex $v$. The (capacity) supply provided by $p$ is $s: A \rightarrow \mathbb{R}_{0}^{+}, a \mapsto \sum_{l \in L, a \in l} p(l) \kappa$, where $\kappa$ is some positive constant specifying the number of passengers that can be transported per vehicle. $p$ is feasible (for the demand $d$ ) if it is balanced and if $s(a) \geq d(a)$ for every $a \in A$. A feasible line plan $p$ is optimal if it minimizes the overall cost $C:=\sum_{l \in L: p(l)>0} c^{f}(l)+\sum_{l \in L} c_{l}^{o} p(l)$, where $c^{f}: L \rightarrow \mathbb{R}_{0}^{+}$denotes a fixed setup cost for each line, and $c^{o}: L \rightarrow \mathbb{R}_{0}^{+}, l \mapsto \sum_{a \in l, a \in A} c(a)$ the operational cost per vehicle.

The demand coverage model can be formulated as an integer program as follows. Let $x_{l} \in\{0,1\}$ be a binary variable that takes value one if line $l \in L$ is selected, and $v_{l}$ an integer variable that defines how many vehicles are operating line $l$.

$$
\begin{aligned}
\min \sum_{l \in L} c_{l}^{f} x_{l}+\sum_{l \in L} c_{l}^{o} v_{l} & \\
\sum_{l \in L_{a}} \kappa v_{l} \geq d_{a} & \forall a \in A, \\
M x_{l}-v_{l} \geq 0 & \forall l \in L, \\
\sum_{l \in L} v_{l} \leq V & \\
x_{l} \in\{0,1\} & \forall l \in L, \\
v_{l} \in \mathbb{N} & \forall l \in L .
\end{aligned}
$$

$\left(\mathrm{DCM}_{\mathrm{MIP}}\right)$

Inequalities (1) of program (DCM $\left.\mathrm{MIP}_{\mathrm{P}}\right)$ make sure that the demand $d_{a}$ on each segment $a \in A$ is covered by the capacity of the set $L_{a}$ of lines that contain $a$. The $v$-variables are coupled with the $x$-variables via inequalities (2) in order to assure that only chosen lines are assigned a positive number of up to $M$ vehicles each. Constraint (3) limits the overall number of vehicles. The objective of program (DCM $\left.\mathrm{MIP}_{\mathrm{P}}\right)$ models both the minimization of fixed and operational costs. 


\section{Unimodality}

By examination of the passenger data for the Istanbul Metrobüs system it becomes apparent that, for any given time, the distribution of the passenger demand volume per station is approximately unimodal: More centrally located stations experience higher traffic. Under these conditions, and if the fixed costs $c^{f}$ are negligible, an optimal line plan is unimodal as well, i.e., for any two lines, one is contained in the other. This greatly reduces the complexity of the problem and allows to solve it in linear time.

Denote by $\bar{a}$ the reverse of $\operatorname{arc} a \in A$, and by $\left.d^{\leftrightarrow}(a):=\max \{d(a), d(\bar{a}))\right\}$ the maximum demand on $a \in A$ in both directions. A line plan $p$ is unimodal if $p(l) p\left(l^{\prime}\right)=0$ for any two forward lines $l$ and $l^{\prime}$ that do not satisfy $[\alpha(l), \omega(l)] \supseteq\left[\alpha\left(l^{\prime}\right), \omega\left(l^{\prime}\right)\right]$ or $\left[\alpha\left(l^{\prime}\right), \omega\left(l^{\prime}\right)\right] \supseteq[\alpha(l), \omega(l)]$, and similar for backward lines.

Theorem 1. If the maximum demand is unimodal and the fixed costs are zero, then there is an optimal unimodal line plan.

Proof. It is easy to see that every balanced line plan $p$ satisfies $s(a)=s(\bar{a})$ for all $a \in A$. Therefore, $p$ is feasible if and only if $s(a) \geq d^{\leftrightarrow}(a)$ for all $a \in A^{\rightarrow}$, i.e., we can restrict our attention to $A \rightarrow$.

Call two forward arcs equivalent if they are not separated by a terminal. Then any two equivalent arcs $a \sim a^{\prime}$ are always assigned the same capacity supply by every line plan, because they are covered by the same set of lines. For any forward arc $a \in A^{\rightarrow}$, let

$$
s_{*}(a):=\max _{a^{\prime} \sim a} d^{\leftrightarrow}\left(a^{\prime}\right) \quad \text { and } \quad k_{*}(a):=\left\lceil s_{*}(a) / \kappa\right\rceil
$$

Then any feasible line plan must provide a capacity supply of at least $s_{*}(a)$ on $a \in A^{\rightarrow}$, and use at least $k_{*}(a)$ vehicles. The unimodality of $d^{\leftrightarrow}$ implies unimodality of $s_{*}$ and, in turn, of $k_{*}$.

It is easy to construct a unimodal line plan $p$ on $A^{\rightarrow}$ with supply $s=s_{*}$, using exactly $k_{*}(a)$ vehicles on any arc. Namely, let $l_{1, n}$ be the line from $t_{1}=1$ to node $t_{m}=n$, set

$$
p_{*}\left(l_{1, n}\right):=\min \left\{k_{*}(1,2), k_{*}(n-1, n)\right\},
$$

subtract $p_{*}\left(l_{1, n}\right)$ from $k_{*}(a)$ for all $a \in A^{\rightarrow}$, and remove all arcs such that $k_{*}(a)=0$; then iterate until all arcs have been removed. Proceed in the same way for $A^{\leftarrow}$.

The resulting line plan is unimodal, feasible, and also optimal, because its cost

$$
C=\sum_{l \in L} p(l) \sum_{\substack{a \in l \\ a \in A^{\rightarrow}}} c(a)=\sum_{a \in A^{\rightarrow}} \frac{s(a)}{\kappa}=\sum_{a \in A^{\rightarrow}} \frac{s_{*}(a)}{\kappa}=\sum_{a \in A^{\rightarrow}} k_{*}(a)
$$

is minimal by minimality of $k_{*}$. 


\section{The Multiperiod Case}

The case at hand, the Istanbul Metrobüs system, is characterized by a highly fluctuating and asymmetrical demand caused primarily by commuters. In the morning hours a high volume of passengers needs to be transported from the periphery to the center, while the flow is reversed in the evening hours. In between the demand falls sharply. Figure 2 gives a more detailed impression of the passenger demands in both directions. In the figure, the $\mathrm{x}$-axis accounts for time and the $\mathrm{y}$-axis for the corresponding demand. The peak-times stand out, and there is a relatively constant demand in between. At any point in time, the spatial demand distribution is very close to being unimodal.

We identify the peak and the off-peak time intervals as follows. Running the demand covering model (DCM $\mathrm{MIP}_{\mathrm{P}}$ ) for every hour from 6 to 23 o'clock produces 18 line plans, represented by their $p$-vectors. We check these vectors for similarities using the classical $k$-means clustering algorithm [3]. Fig. 3 shows the results for $k=2$ : The algorithm clusters the time windows from 7 to 9 and 17 to 20 together, which correspond to the peaks. All other time windows form a common off-peak section.

Two line planning strategies that exploit this temporal subdivision suggest themselves: On the one hand it might be advisable to run separate schedules during peak and off-peak times to cope with the different demands. On the other hand, the peak demands exceed the base demands. It might therefore be possible to run a continued base line plan throughout the entire day, augmented by additional resources dur-

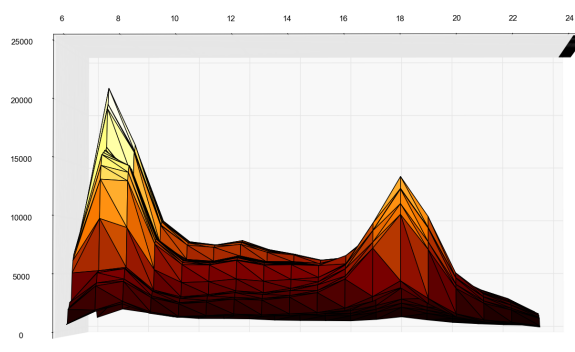

(a) Demands in direction from east to west.

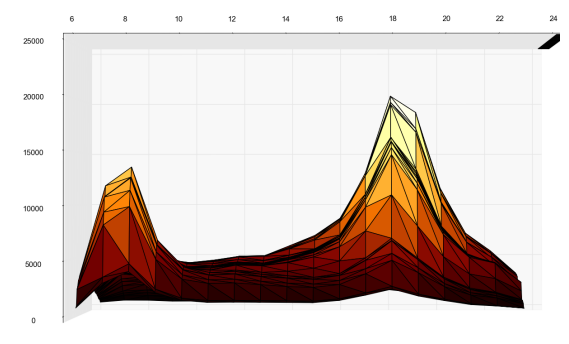

(b) Demands in direction from west to east.

Fig. 2 Traffic demand in the Istanbul Metrobüs system over 24 hours, summed over all OD pairs.

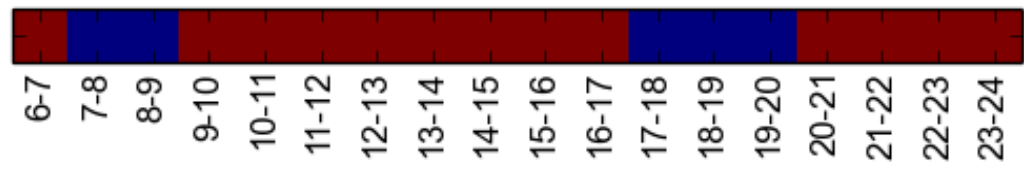

Fig. 3 Identifying peak and off-peak times by clustering hourly line plans. 
ing peak times. Such an augmentation can be seen as an analogon over time of a unimodal line plan, which can be seen as a capacity augmentation in space. Our theoretical findings on unimodal line plans suggest that resorting to unimodal line plans is feasible for almost unimodal demands. In combination with a similar result over time, this would make a good case for this traditional planning procedure.

We consider the following two computational scenarios in a setting without fixed costs and operational costs based on driven distance:

(1) a discontinued base schedule with independent peak time line plans and

(2) a continued base schedule with peak time line plans on top.

Computations were performed on a Intel i7-4790 3.60GHz CPU using CPLEX 12.6 as a MIP solver.

\begin{tabular}{l|r|r|r|r}
\hline \multirow{2}{*}{ Scenario } & \multicolumn{2}{|c|}{ MIP solution } & \multicolumn{2}{c}{ unimodular solution } \\
& value [km] & computation time & value [km] & computation time \\
\hline \multirow{2}{*}{$(1)$} & 12041 & $1.9 \mathrm{~s}$ & 12056 & $0.9 \mathrm{~s}$ \\
$(2)$ & 12144 & $1.8 \mathrm{~s}$ & 12164 & $0.8 \mathrm{~s}$ \\
\hline
\end{tabular}

Table 1 Comparing different line planning approaches for the Istanbul Metrobüs on weekdays.

The computations corroborate our expectations that service augmentation in space and time produces results whose quality is on a par with unrestricted planning w.r.t. operational costs.

\section{References}

1. Borndörfer, R., Grötschel, M., Jaeger, U.: Planning problems in public transit. Production Factor Mathematics pp. 95 - 122 (2010). DOI 10.1007/978-3-642-11248-5

2. Liebchen, C.: Linien-, Fahrplan-, Umlauf- und Dienstplanoptimierung: Wie weit können diese bereits integriert werden? In: Heureka'08. Stiftung Heureka, FGSV Verlag (2008)

3. McCallum, A., Nigam, K., Ungar, L.H.: Efficient clustering of high-dimensional data sets with application to reference matching. In: Proceedings of ACM, KDD '00, pp. 169-178. ACM, New York, NY, USA (2000). DOI 10.1145/347090.347123. URL http:// doi . acm.org/ $10.1145 / 347090.347123$

4. PTV AG: http://vision-traffic.ptvgroup.com/en-us/products/ ptv-visum/

5. Torres, L.M., Torres, R., Borndörfer, R., Pfetsch, M.E.: Line Planning on Paths and Tree Networks with Applications to the Quito Trolebus System. In: M. Fischetti, P. Widmayer (eds.) ATMOS'08, OpenAccess Series in Informatics (OASIcs), vol. 9. Schloss Dagstuhl-LeibnizZentrum fuer Informatik, Dagstuhl, Germany (2008). DOI http://dx.doi.org/10.4230/OASIcs. ATMOS.2008.1583. URL http://drops.dagstuhl. de/opus/volltexte/2008/ 1583 